\title{
STUDIES IN PLANTAR ULCERS IN LEPROSY
}

\section{ETIOLOGY OF PLANTAR ULCERS}

\author{
E. W. Price, F.R.C.S. \\ Orthopaedic Surgeon to Leprosy Service, Eastern Nigeria
}

\section{Introduction}

The problems of plantar ulcer in leprosy include why it occurs in the first place, why it resists simple treatment, and why it often recurs after healing. At the moment we do not know the answer to any of these questions, though there have been several suggestions on which different types of treatment have been based.

In this paper we summarise the known facts about plantar ulceration in leprosy and then discuss various theories of their causation, and finally suggest a modified etiology which corresponds to clinical observations and which will serve as a basis of treatment, and protection against relapse.

Similar ulcers occur in the neuropathic foot of diabetes, though in this there is often a vascular disturbance which is absent in leprosy. OAtLEY, et al. (1956)1 summarised current views on the etiology of these diabetic lesions of the feet, and gave clinical photographs of plantar ulcers which appear identical with those of leprosy.

\section{Accepted Facts about Plantar Ulcers in Leprosy}

1. The ulcer occurs on an anaesthetic sole

The skin on which a plantar ulcer occurs is always anaesthetic. The relation of ulceration to deep sensation is less clear. Deep ulcers may be either painful or painless, and surgical intervention can sometimes be undertaken in the deep tissues without the induction of anaesthesia.

We studied a recent series of cases in an attempt to correlate the occurrence of plantar ulcers with the state of deep sensation, using the presence or absence of joint sensation as an indicator of the integrity of deep sensibility. The subject appreciates the position of a joint by means of sensation transmitted from the joint itself and from the skin, and sensory mechanisms from tendons and muscles play no part in it (HARRISON, 19582). Nevertheless, some of our patients obviously did estimate the position of the joint by contracting the flexor and extensor muscles in alternation, and calculating the position of the joint from the varying tensions. However this is not true joint sense. We used the big toe to test the first metatarso-phalangeal joint, because the toe is easy to handle, and plantar ulcers are common over this joint. We had also noted that normal barefooted villagers are aware of the position of the big toe when they are not always clear about the position of their lateral toes. 
We tested 50 consecutive cases in this way, and found no correlation between the presence of ulceration over the first metatarsophalangeal joint and the presence or absence of position-sense in the joint itself. However similar observations on a series of new ulcers might reveal significant facts. The present series included cases of varying duration, and it may well be that joint-sense was absent at the period when the ulcer occurred. It is also possible that deep sensation may be absent in soft tissues when joint-sense is intact. which is a relation difficult to establish clinically. We have found no references in the literature to observations of this sort, and it calls for investigation by those who deal with such cases.

2. Some anaesthetic soles never ulcerate at any period

This fact is related to the previous one, and is stated separately because study of deep sensation in non-ulcerated soles may help us to understand the problem, and will play a part in plans to provide suitable footwear for the protection of anaesthetic feet in leprosy patients. It would be a most helpful advance if we could know which feet are likely to ulcerate and which not.

3. Ulcers occur on the pressure-hearing areas of the sole

This is true of the undeformed foot. In a foot mutilated by advanced infection or by surgical intervention, ulceration will occur wherever the pressures are maximal. Apart from these latter cases, all plantar ulcers can be plotted on a tracing of the walking footprint (see drawings in Fig. 2 of the Mechanics of the Foot in Relation to Plantar Ulcers, Lep. Rev. 30, 2: A pril, 1959, facing p. 101). This does not apply to the standing footprint, in which the pressure areas include only the heel and metatarsal pads. The tips of the toes may leave an imprint, but in the standing foot are not pressure-bearing areas, nor is the area under the interphalangeal joint of the big toe. Both these latter are common sites for plantar ulceration.

4. A Plantar ulcer will heal with hed rest, or if immobilized in a walking plaster cast

It has long been known that plantar ulcers will heal if the patient is kept off his feet long enough, and periods of four to six weeks are adequate in uncomplicated cases. It is also known that they will heal if the foot is encased in a walking plaster cast, taking a week or two longer but not requiring complete rest in bed. If plaster cast immobilization is kept up long enough, all ulcers will heal eventually without surgical intervention, though for feet grossly affected period up to one year may be needed.

The earliest reference to treatment by plaster casts appears to be that of KHAN (1939) ${ }^{3}$, and repeated mention has been made of its success since then, (Milroy PAUl, 19474, Fisher, 19555, BoSE, 19566). There is a reluctance to adopt the method, which is undoubtedly due to the high proportion of relapses when natural walking is resumed. It has been suggested that the healing achieved in a plaster cast is due 
to the distribution of pressure over the full area of the sole by a closely-fitting cast. However, experience of such casts makes one doubtful whether they really do this for long, for most are quite loose at the end of a month and can hardly be distributing weight. In spite of this, the ulcer heals and it seems that some other process promotes it.

\section{A plantar ulcer overlies a bony prominence}

Plantar ulcers are found over the following prominences in the unmutilated foot; the metatarsal heads; the tubercle on the base of the 5th metatarsal; the head of the proximal phalanx of the big toe; the tubercles of the calcaneus; the heads of the distal phalanges of the toes.

It should be noted that the tubercle on the base of the 5 th metatarsal is a lateral projection in the normal foot, and only presents in the sole if there is weakness in the pronators which evert the foot. The head of the proximal phalanx of the big toe and the tips of the toes only become bony prominences in the walking foot.

Study of the mechanics of the foot makes it more likely that the operative factor is not so much the presence of the bony prominence but the points of pressure-rotation during the walking roll (see Leprosy Review', 30, 2, April, 1959, p. 102.)

\section{Theories of the Etiology of Plantar Ulcers}

The literature does not include careful studies into the etiology of plantar ulcers in leprosy, and etiological theories have to be deduced from the type of treatment suggested. These theories include the following:

1. That plantar ulcers are specific lesions

Though this has not been explicitly stated anywhere, the local use of specific drugs such as chaulmoogra oil or deratives of DDS implies a suspicion of it.

In the earlier experiments of Lowe et al. (1936)7, hydnocarpus oil was injected weekly into the base of the ulcer by a series of punctures, and photographs showed that the ulcer healed in six weeks. However, ulcers will heal spontaneously if weight-bearing is avoided for six weeks, and the beneficial effects may well have been due to the fact that walking on the ulcer was made difficult or impossible by this treatment. The report stated that all ulcers do not respond to injection treatment.

In a later series by Dharmendra, et al. (1955) ${ }^{8}$ a derivative of DDS was used locally in fifteen cases, with X-ray controls. Healing occurred in only nine of these cases after treatment from 1 to 12 months, with an average of nine months. Judging by the clinical descriptions, the time needed for healing is no less, and appears longer, than that associated with simple rest or immobilization in a walking cast. 
Thus there is little probability that plantar ulcers are specific lesions.

2. That the autonomic nervous system is involved

Some workers have wondered whether lesions of the autonomic nervous system contribute either to the occurrence of plantar ulcer or to its chronicity, with particulat reference to disturbance of the blood supply to the part. Treatment based on this surmise would take note of the effect of a damaged autonomic system, or seek to increase the blood supply to the ulcer-bearing area in a normal autonomic system.

In the treatment of tropical ulcers in pre-antibiotic days there was some success from increasing the blood supply in this way (PRICE, 1945)9. There are few records of the use of this method in ulceration in leprosy. VISHNEVSKY $(1938)^{10}$ reported a series treated by novocaine blockade, but concluded that the results were indefinite. LEITNER (1938) ${ }^{11}$ prepared arteriograms of feet amputated for long-standing ulceration in leprosy and found an ample blood supply even after long-standing infection and advanced destruction.

As in the strapping treatment for varicose ulcers the success of plaster casts might be due to support given to the peripheral circulation. To test this we treated a few cases of plantar ulcers by giving them a plaster sandal to wear, which did not reach above the ankle. The sandal had to be strapped to the foot to prevent it coming off, and it was difficult to avoid rubbing the heel, but the ulcers healed in much the same time as those which had long casts. It seems as if disturbance of the autonomic system plays little part in the pathology of plantar ulceration.

3. That trauma is the prime cause

Infected trauma can cause an ulcer on the sole, as on the hand or elsewhere on the body skin, but the lesion can be recognized as an infected cut or puncture in most cases, and we think it can be clearly distinguished from the chronic ulceration with which we are concerned here. However, Cochrane (1947) states "We believe all trophic ulcers commence from injury; this may be so slight as hardly to be noticed." He gives the impression that the trauma concerned is damage to the skin by an external agent. BRAND (1950) ${ }^{12}$ concludes that "The two factors which produce ulceration are sustained pressure and active injury. In general, people who wear shoes suffer from sustained pressure and people who walk barefoot from direct injury."

In order to study the relation of external trauma to plantar ulcers the writer examined every week for six months the feet of 500 patients in a leprosarium, aided by a trained Nigerian assistant. We paid particular attention to the occurrence of trauma by cuts and pricks, etc., and to cases in whom plantar ulceration obviously impended. We confirmed Cochrane in his observation that the 
earliest lesions are frequently in the form of idiopathic blisters, but there seemed to be no connection between obvious trauma and subsequent plantar ulcer. The idea that the trauma may be so small as to escape careful regular examination also seemed wide of the mark, for the blisters were observed to arise from deep tissues and follow a sequence at a time when in very many cases the skin surface was perfectly intact.

The relation of superficial trauma to plantar ulcer is not merely academic. If such trauma were really the initial lesion it would be logical to provide protective footwear for all leprosy patients who have anaesthetic soles. Those who have experience in providing and maintaining such footwear for even a limited number of patients in a leprosarium, will realise the amount of work and expense involved; it would be even more difficult in rural clinics. On the other hand, if external trauma only plays a minor part, the provision of footwear can be limited to those patients who are passing through the phase when plantar ulcer is a danger. We believe that this period can be judged by the weekly palpation of the ulcer-bearing areas of anaesthetic soles. Only these cases are in need of protective footwear.

\section{That plantar pressure is a cause of plantar ulcer}

That the pressure of the weight of the body is important in producing plantar ulcers is clear, but it is not so clear how it acts. BRAND (1950)12 believes that prolonged standing is the dangerous factor, stating "It is fundamental that no patient be allowed to stand for more than five minutes at a time". In this connection we recall that pressure ulcers from the recumbency of sleep, where the weight of the limb rests on the posterior aspect of the heel or one on of the malleoli, are remarkably uncommon in leprosy. It is also significant that the pressure distribution during standing in patients with anaesthetic soles varies rapidly from one part of the sole to another as the patient maintains his balance without the help of the sensory mechanism of the sole. In this the muscles of the calf and front of the lower leg are in constant movement in order to maintain balance.

\section{That clawing of the toes exposes the metatarsal heads}

It is an attractive theory that intrinsic muscle weakness is a factor in the production of plantar ulceration, even though perforating ulcers have been reported in tabes, where muscle weakness is not a feature. Because of the suggestion that the cocked toes of leprosy expose the metatarsal heads to pressure and injury, and so predispose to ulceration, we kept a careful check on patients with clawed or cocked toes in relation to the incidence of ulcer on the corresponding metatarsal pad. We soon found that there was no obvious relation between the two. We also examined a group of healthy subjects in a training school and found a surprising number of normal feet which had toes which did not touch the ground on standing. 
We think therefore that clawing of the toes has no relation to plantar ulcer insofar as it exposes the metatarsal head. Often a foot with no toes is remarkably free from ulcers. We hold strongly that the inability of weakened toes to perform their protective function at the moment of the walking roll is a potent factor in the production and chronicity of plantar ulceration in leprosy.

Suggested Etiology as a Basis for the Treatment and Prevention of Plantar Ulcers

Thus a critical examination of existing theories reveals several unsatisfying features, if only because treatment based on them has yielded no lasting results so far. No theory fits all the facts, when one carefully examines a series of anaesthetic feet. We suggest the following etiology which incorporates the known facts, adds recent observations, and gives a plan of treatment and prophylaxis which can give good results. It also gives an indication of the type of footwear likely to be of value, and the circumstances in which protective footwear should be provided.

The factors involved in the production of plantar ulcer are:

1. An anaesthetic foot: superficial anaesthesis is invariable, but deep anaesthesia appears to be variable and may be absent at the moment of ulceration:

2. A walking foot: patients who do not walk do not develop ulcers and they occur at points on the sole where there is pressurefriction round points of rotation of the walking roll; these include the point of contact at the heel, the points of dorsiflexion of the foot at the mid-lateral and the metatarso-phalangeal joints, and the points of push off at the tips of the toes:

3. A foot with a damaged intrinsic musculature: weakness of this nature compromises the mechanism by which the points of rotation are protected from damage during walking.

It follows from the above that plantar ulcers will heal and remain healed if the walking roll is interrupted. This is ensured if the patient is in bed, if he is wearing a plaster cast even if walking in it, and if he wears footwear with a rigid sole; such footwear also protects the points of contact at the heel and in push off at the toes. Later we hope to report the results of a series of cases treated for a year by this method.

\section{Summary}

The accepted facts about plantar ulcers in leprosy are that they occur on an anaesthetic sole, though some anaesthetic soles never ulcerate at any time; that ulcers develop on the pressure-bearing areas of the sole; that they will heal with bed rest, or if immobilized in a walking plaster cast; that they overlie bony prominences.

The theories of the etiology of plantar ulcers are unsatisfactory, and include the ideas that plantar ulcers are specific lesions, that the 
autonomic nervous system is involved, that trauma is the prime cause, that plantar pressure is a cause, and that clawing of the toes exposes the metatarsal heads. From his experimental studies the author thinks that plantar pressure is the most valid of these, but linds that it is not so much standing pressure as the inability of weakened toes to perform their protective function at the moment of the walking roll.

He gives a suggested etiology for plantar ulcers which can be used for prevention and treatment. The chief factors in the production of them are an anaesthetic foot, a walking foot, a foot with damaged musculature. Plantar ulcers will heal and remain healed if the walking roll is interrupted, either by bed rest, or by wearing a plaster cast, or by wearing footwear with a rigid sole. He hopes later to report the results of a series of cases treated for a year on these principles.

\section{References}

1. Oatley, W., Caterall, R. C., and Martin, M. M. Brit. Med. J. 1956, 2, 953

2. Harrison, R. J. Report of B.M.A. Meetings, Brit. Med. J., July, 1958, 26, 230.

3. KHAN, J. S. Leprosy in India, 1939, 11, 19.

4. Milroy Paul, quoted by Cochrane, Practical Textbook of Leprosy, Oxford Univ. Press, 1947.

5. FISHER, C. Leprosy' Review, 1955, 26, 107.

6. Bose, D. N. Leprosy in India, 1956, $28,77$.

7. Lowe, J. and ChatterJI, S. N., Leprosy in India, 1936, 9, 115.

8. Dharmendra, ChatterJi, S. N., and Sen, N. R. Leprosy in India, 1955, 27, 180.

9. Price, E. W. Transact. Roy. Soc. Trop. Med. and Hyg., 1945, 39, 83.

10. VISHNEVSKY, S. M. Internat. J. of Leprosy, 1938, 6, 477.

11. Leitner, A. J. Internat. J. of Leprosy, 1938, 6, 471.

12. Brand, P. W. Journ. Christian Med. Assoc. of India, 1950.

\section{Acknowledgement}

I am indebted to the Director of Medical Services, Eastern Nigeria, for permission to publish. 